\title{
Longitudinal dynamics in a Lennard-Jones binary liquid: Crossover from hydrodynamics to the molecular regime
}

\author{
T.Bryk, I.Mryglod \\ Institute for Condensed Matter Physics of the National Academy of \\ Sciences of Ukraine, 1 Svientsitskii Str., 79011 Lviv, Ukraine
}

Received February 21, 2004

Longitudinal collective dynamics of an equimolar Lennard-Jones KrAr mixture is studied in detail in a wide range of spatial and time scales. Combining both the molecular dynamics simulations and analytical generalized collective mode approach, we calculated the spectrum of generalized collective excitations and analyzed the dominant dynamic processes that determine the main contributions to time correlation functions in different regions - starting from the hydrodynamic limit and up to the range of the so-called molecular regime. The origin of collective propagating modes as well as the specific features of their dispersion laws within and beyond the hydrodynamic region are established. It is shown that the structural relaxation and processes, connected with mutual diffusion of particles, determine mainly the central peak of total dynamic structure factor beyond the hydrodynamic region. The dispersion laws, obtained for the propagating modes in our analytical approach, are compared with the dispersion curves, estimated from the maxima positions of partial current spectral functions. The difference in these two sets of numerical results is discussed.

Key words: collective dynamics, binary mixture, collective excitation, structural relaxation

PACS: 05.20.Jj, 61.20.Ja, 61.20.LC

\section{Introduction}

Collective dynamics of liquids is one of the important and yet unresolved problems of modern statistical physics. Its complexity is mostly connected with very complicated interplay of various dynamic processes on different spatial and time scales that cannot be reproduced within simplest analytical theories. Only in hydrodynamic limit, when the slowest processes are well separated in time scale from the fast kinetic ones and a liquid could be treated as a continuum without any molecular 
structure, it is possible to obtain relatively simple and working expressions for the hydrodynamic time correlation functions $[1,2]$.

Rapid development of computers in the last two decades has made it possible to perform computer simulations for systems with large numbers of particles, enabling us to study in detail different time correlation functions in a wide range of spatial and time scales - from hydrodynamic limit and up to molecular regime, where the microscopic processes sufficiently affect the form of time correlation functions. Even in the case of simple liquids we can feel the lack of a reliable theoretical approach to the description of collective processes in liquids on a molecular scale. For instance, only recently [3-6] it was realized that the structural relaxation plays the dominant role beyond the hydrodynamic region making the leading contribution to the central peak of dynamic structure factor $S(k, \omega)(k$ and $\omega$ being wavenumber and frequency, respectively), while in long-wavelength limit the most important contribution is caused by the hydrodynamic relaxation process of thermal diffusion. Therefore, one can speak about a crossover phenomenon in the liquid dynamics, describing the change of dominant dynamic processes when spatial and time scales vary from hydrodynamic region to molecular regime.

For binary liquids, the dominant dynamic processes that determine the collective dynamics beyond the hydrodynamic region are still under discussion. For the longitudinal dynamics, it is generally accepted that there exist at least two branches of propagating excitations beyond the hydrodynamic region, which in a shortwavelength region describe the partial dynamics of heavy and light components in a binary mixture. However, regarding the dispersion laws of these two branches by approaching the hydrodynamic region there is no clear model for describing the crossover in dispersion from the molecular region to the hydrodynamic one. Especially big problems arise regarding mixtures composed of disparate mass particles. So far it has not been finally established what is going on with the high-frequency propagating branch in the long-wavelength limit. Hence, the problem of "fast sound" [7] reported in the literature over almost 20 years is still far from being finally solved. It is not also known what is the role of concentration in the damping of high-frequency branch, how the mass ratio affects the width of hydrodynamic region, how will the dispersion law change by decreasing the concentration of relevant species up to the impurity limit.

One of the most natural ways of exploring the collective dynamics in binary liquids is to vastly apply simple theoretical models and reliable schemes for the analysis of computer experiments in order to clarify the dominant relaxing and propagating processes in a wide range of wavenumbers $k$ and frequencies $\omega$, including the hydrodynamic limit. A good choice for such a study is an equimolar binary liquid with relatively small mass ratio that would allow one to compare the obtained results with those known for simple fluids. In such a way one can systematically target further the effect of concentration or mass ratio onto the dispersion laws for propagating excitations. Recently, the main dynamic processes in the transverse dynamics of a binary equimolar KrAr liquid $[8,9]$ were studied by combining the molecular dynamics (MD) simulations and an analytical generalized collective modes (GCM) approach 
[10-12]. A clear picture of transverse dynamics in terms of two branches of propagating excitations was established: in short-wavelength region the branches show a well observed partial character, describing the dynamics of light and heavy components, while in the long-wavelength region the low-frequency branch exhibits the collective shear-wave dispersion with a propagation gap in $k \rightarrow 0$ limit and the high-frequency branch corresponds to the pair of collective optic-like excitations. A simple theoretical model for separated transverse mass-concentration current fluctuations allowed us to explain the origin of optic-like branch in the long-wavelength limit and to clarify a physical mechanism causing the damping of transverse optic-like excitations $[8,9]$. Later, a similar three-variable model for the dynamics of mass-concentration fluctuations was solved for the longitudinal case [13]. Remarkably, exactly the same mechanism of damping for the optic-like excitations was found in the case of the longitudinal dynamics. In particular, it was shown that high mutual diffusion and a tendency to demixing, when the atoms are mainly surrounded by like particles, cause strong damping and can even suppress the optic-like branch. Such a picture seems to work in a gaseous mixture $\mathrm{He}_{0.65} \mathrm{Ne}_{0.35}$ [14,13], where the high-frequency branch vanishes from the spectrum in the small- $k$ region because of the strong effect of mutual diffusion processes.

Thus, on the one hand, the analysis of collective modes spectrum obviously indicates that in the long-wavelength region there commonly exists an optic-like branch of mass-concentration waves unless some specific processes in the liquid (like high diffusion or a tendency to demixing) can suppress it because of strong dissipation. This last statement can be rigorously proved by neglecting the dissipation terms in the relative spectral problem. On the other hand, in the literature there are many reports on the dispersion curves in binary liquids, obtained numerically from the estimates of peak positions in the MD-derived partial current spectral functions $C_{\alpha \alpha}(k, \omega), \quad \alpha=A, B$ [15-18]. The general picture, found in these studies for the dispersion curves of propagating modes, is as follows: two branches, well defined in the short-wavelength region and describing the high- and low-frequency excitations, are merged into a single branch with linear sound-like dispersion in small $k$ limit. This point will be discussed in detail in this paper by applying both the GCM approach and numerical estimates method and by analyzing the results obtained.

In fact, hydrodynamic equations reflect the local conservation laws for dynamic variables, connected with additive integrals of motion. For a binary liquid they are [19] the densities of total number of particles $n_{t}(k, t)$, concentration $c(k, t)$, total momentum $\mathbf{J}_{t}(k, t)$, and energy $\varepsilon(k, t)$, respectively. Solution of the hydrodynamic equations describes the slowest dynamic processes on large spatial scales which correspond in the case of binary liquids to the thermal and concentration diffusion (two relaxing processes), and a pair of sound propagating modes (propagating processes). In terms of relative eigenvalues for small wavenumbers $k$ one can write down

$$
d_{\alpha}(k)=D_{\alpha} k^{2}, \quad \alpha=\mathrm{h}, \mathrm{c}
$$

for the heat and concentration diffusive modes with $D_{i}$ being the damping coefficients, and

$$
z_{\mathrm{s}}=\Gamma_{\mathrm{s}} k^{2} \pm \mathrm{i} c_{\mathrm{s}} k
$$


for sound propagating modes, where $\Gamma_{\mathrm{s}}$ and $c_{\mathrm{s}}$ denote the sound attenuation coefficient and sound velocity, respectively. These hydrodynamic eigenvalues are responsible for $k$-dependence of the dynamic structure factor $S(k, \omega)[1,19]$, which can be written as follows:

$$
\begin{aligned}
\frac{S^{\text {hyd }}(k, \omega)}{S(k)}= & \mathcal{G}_{\mathrm{s}}^{n n} \sum_{\alpha=+,-} \frac{\Gamma_{\mathrm{s}} k^{2}-\alpha k\left(\omega / c_{\mathrm{s}}+\alpha k\right) b_{n n}}{\left(\omega+\alpha k c_{\mathrm{s}}\right)^{2}+\left(\Gamma_{\mathrm{s}} k^{2}\right)^{2}} \\
& +\sum_{\alpha=h, c} \mathcal{G}_{\alpha}^{n n} \frac{D_{\alpha} k^{2}}{\omega^{2}+\left(D_{\alpha} k^{2}\right)^{2}}
\end{aligned}
$$

and consists of two central Lorentzians with the amplitudes $\mathcal{G}_{\mathrm{h}}^{n n}$ and $\mathcal{G}_{\mathrm{c}}^{n n}$ that appear due to the heat and concentration diffusion, respectively, and two non-central Lorentzians (so-called Brillouin peaks located at $\omega \simeq \pm c_{\mathrm{s}} k$ ) with amplitude $\mathcal{G}_{\mathrm{s}}^{n n}$. Although the expression (3) is valid only in hydrodynamic limit, it is widely used for the analysis of experimental data and the results of MD simulations. One can see that this expression contains no indication of the appearance of optic-like propagating modes. This is because only the slowest processes are usually taken into account within the hydrodynamic treatment, and the additional terms in (3) that can be caused by faster phenomena are just neglected [they contribute in higher order with respect to $k$ and/or $\omega$ ]. It will be seen later that the optic-like modes give a good example of this kind of processes.

The goal of this study is to investigate the main propagating and relaxing processes that dominate in different spatial and time scales in the longitudinal dynamics of a binary equimolar liquid with a relatively small mass ratio. This allows us to clarify some key-points being under discussion in the literature, namely: (i) to establish the physical origin of collective modes formation in different regions of wavenumbers, starting from the hydrodynamic one, and (ii) to analyze their contributions to the dynamic structure factors and spectral current functions depending on spatial and time scales considered.

The remaining part of the paper is organized as follows. In section 2 we describe the details of our MD simulations and report generalized thermodynamic quantities and time correlation functions of Lennard-Jones KrAr mixture. In section 3 we discuss the numerical results found for the generalized collective modes within several theoretical schemes in order to justify the origin of mode formation and to establish the dynamic processes with dominant contributions in different regions of $k$ considered. Section 4 contains our conclusions of this study.

\section{Static and time correlation functions}

\subsection{Some introductory definitions}

Let us start from the definition of dynamic variables of total $n_{t}(k, t)$ and massconcentration $n_{x}(k, t)$ densities,

$$
n_{t}(k, t)=n_{1}(k, t)+n_{2}(k, t),
$$


and

$$
n_{x}(k, t)=\frac{m_{1}}{\bar{m}} x_{2} n_{1}(k, t)-\frac{m_{2}}{\bar{m}} x_{1} n_{2}(k, t),
$$

for a binary system of $N$ particles, $N=N_{1}+N_{2}$, with atomic masses $m_{1}$ and $m_{2}$, respectively, where

$$
n_{\alpha}(k, t)=\frac{1}{\sqrt{N}} \sum_{i=1}^{N_{\alpha}} \mathrm{e}^{\mathrm{ikr} \mathbf{r}_{i}^{\alpha}}, \quad \alpha=1,2,
$$

are the ordinary partial densities. The notations for reduced mass $\bar{m}=\left(N_{1} m_{1}+\right.$ $\left.N_{2} m_{2}\right) / N$ and mass-concentrations $x_{\alpha}=N_{\alpha} m_{\alpha} / N \bar{m}$ were introduced in (5). An advantage of such a definition for the mass-concentration density is its simple relation to the longitudinal mass-concentration current $J_{x}(k, t)$, namely:

$$
\frac{\partial n_{x}(k, t)}{\partial t}=\frac{\mathrm{i} k}{\bar{m}} J_{x}(k, t)
$$

and, as it was shown in $[8,9], J_{x}(k, t)$ is a dynamic variable orthogonal to the total mass-current density $J_{t}(k, t)$. This makes especially convenient the theoretical treatment of dynamic processes in small wavenumbers region in terms of $t-x$ variables, where the collective type of the dynamics prevails (see [13]), while for large wavenumbers the partial character of dynamics is dominant.

\subsection{Details of molecular dynamics simulations}

We have performed MD simulations for an equimolar Lennard-Jones KrAr liquid at temperature $T=116 \mathrm{~K}$ and density $n=0.0182 \AA^{-3}$. The time evolution of basis dynamic variables has been obtained from production runs of standard molecular dynamics simulations in microcanonical ensemble using the system of 864 particles in a cubic box. Regular production run took over $3 \times 10^{5}$ time steps, while for three lowest $k$-values, in order to obtain the desired convergence of relevant static averages and time correlation functions, the system has been simulated over $2.1 \times 10^{6}$ time steps. Twenty two $k$-points were sampled in MD simulations, and the smallest wavenumber reached in MD is $k_{\min }=0.1735 \AA^{-1}$. To reduce the dimension of relevant quantities the following energy, mass, spatial and time scales have been used in our simulations: $\epsilon=k_{\mathrm{B}} T, \mu=\bar{m}, \sigma=k_{\min }^{-1}, \tau=\sigma(\mu / \epsilon)^{1 / 2}=4.598$ ps.

We combined MD simulations with the parameter-free GCM approach for the study of the longitudinal collective mode spectrum, time correlation functions (TCFs) and separated contributions to certain TCFs, caused by various collective excitations. For each sampled $k$-point the shape of MD-derived time correlation functions and the spectrum of eigenvalues have been analyzed within the eight-variable GCM approach with the following basis set of dynamic variables:

$$
\mathbf{A}^{(8)}(k)=\left\{n_{t}, n_{x}, J_{t}, J_{x}, \varepsilon, \dot{J}_{t}, \dot{J}_{x}, \dot{\varepsilon}\right\} \text {. }
$$

Comparing (8) with the standard set of hydrodynamic variables, one can see that the mass-concentration fluctuations are treated with the same precision (with respect to the order of frequency sum rules reproduced exactly) as the total density 


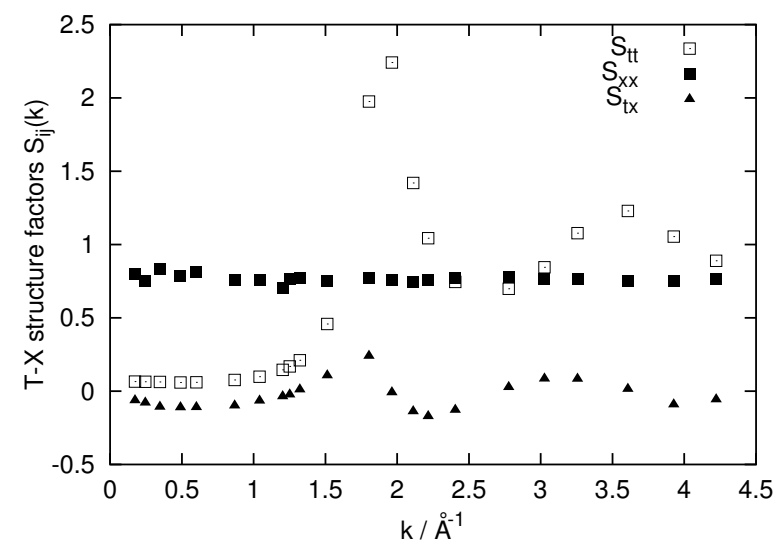

Figure 1. Static structure factors for a Lennard-Jones equimolar KrAr mixture at $116 \mathrm{~K}$ obtained in MD simulations.

fluctuations. Moreover, by applying the set of dynamic variables (8) within the GCM scheme, we provide the high level of treatment for the energy fluctuations (see [12]) (the theoretical energy-energy TCF explicitly reproduces at least first three frequency moments in the whole $k$ domain considered).

\subsection{Static properties}

The numerical results, obtained directly in MD simulations as static averages, for the static structure factors

$$
S_{i j}(k)=\left\langle n_{i}(k, 0) n_{j}^{*}(k, 0)\right\rangle, \quad i, j=t, x,
$$

defined on the total (4) and mass-concentration (5) densities, are shown in figure 1.

One can see in figure 1 the complete difference in the shape of static structure factors $S_{t t}(k)$ and $S_{x x}(k)$. As for simple liquids the total density structure factor $S_{t t}(k)$ behaves in a regular way. It tends to a constant connected with the isothermal compressibility $\kappa_{\mathrm{T}}$ in the long-wavelength limit, and at $k_{\mathrm{p}} \approx 1.9 \AA^{-1}$ this structure factor has a well-defined main maximum. The mass-concentration structure factor $S_{x x}(k)$ in contrast to $S_{t t}(k)$ is almost a structureless function of wavenumber which means almost uniform $A-B$ solubility on different spatial scales. The form of static structure factors $S_{t t}(k)$ and $S_{x x}(k)$ is an important input for the subsequent analysis of dynamic properties.

The $k$-dependence, found for the static correlation function "energy-energy" $S_{e e}(k)$, is plotted in figure 2 (upper frame). This function reflects all the features of $S_{t t}(k)$, while the static cross-correlation functions $S_{e t}(k)$ and $S_{e x}(k)$ (lower frame in figure 2) are negative in the whole $k$-range considered and show a similar oscillating behaviour. Note that $S_{e t}(k)$ displays much more pronounced oscillations than $S_{e x}(k)$.

The generalized hydrodynamic theory usually operates with the so-called generalized thermodynamic quantities (see, e.g., [14]) which are $k$-dependent functions 

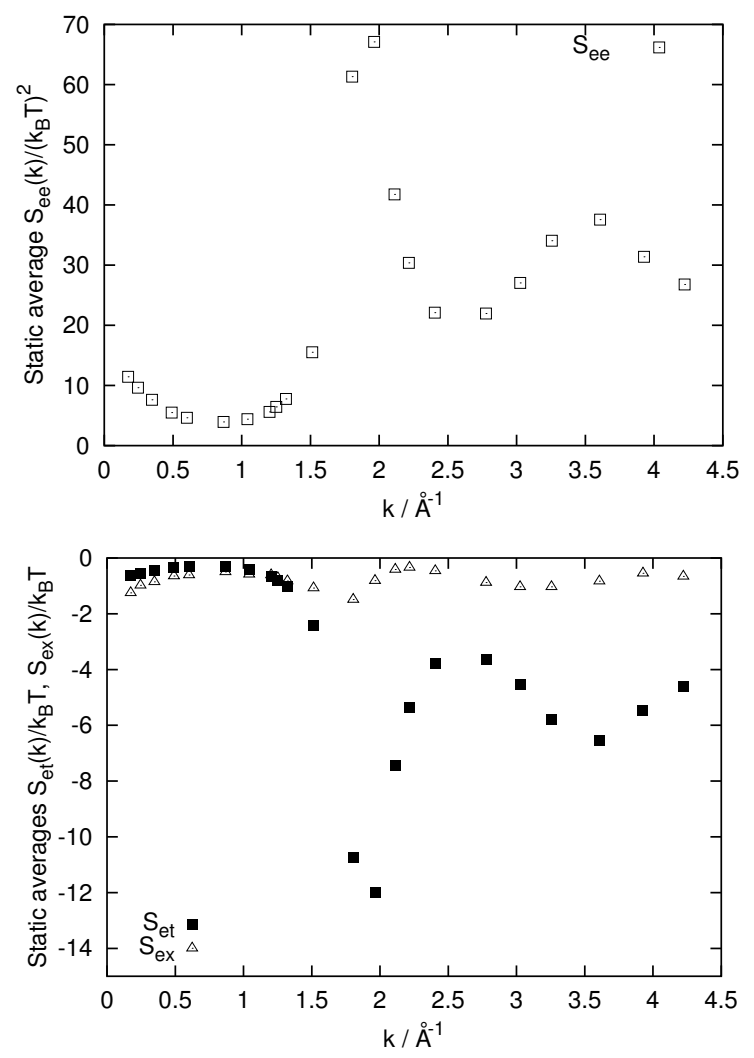

Figure 2. Reduced static correlation functions of a binary liquid $\mathrm{KrAr}$ at $116 \mathrm{~K}$ : (i) energy-energy (on the top); (ii) energy-density and energy-mass concentration (on the bottom).

and in the limit $k \rightarrow 0$ tend to their thermodynamic values observed in real experiments. In figure 3 we show four generalized thermodynamic quantities, namely, the generalized dilatation $\delta(k)$, the normalized linear expansion coefficient $\alpha_{\mathrm{T}}(k) T$, the generalized specific heat at constant volume $C_{\mathrm{V}}(k)$ and the generalized ratio of specific heats $\gamma(k)$, calculated for the system considered in our MD simulations. The expressions for these generalized thermodynamic quantities can be found in [14]. Note, first, that all four generalized thermodynamic quantities in figure 3 have well defined peaks at the position $k_{\mathrm{p}}$ of the main maximum of the total static structure factor. And, second, a lot of useful information can be extracted from such dependencies. In particular, from these calculations, considering small $k$ limit, we can predict the value of $\alpha_{\mathrm{T}}=0.0043 \mathrm{~K}^{-1}$ for linear expansion coefficient of a binary KrAr liquid at the temperature of $116 \mathrm{~K}$.

The generalized $k$-dependent specific heat at constant volume $C_{\mathrm{V}}(k)$ is in agreement with the value of $2.43 k_{B}$ at $k=0$, obtained independently via the fluctuation formula during the MD production runs. The generalized ratio of specific heats tends to a value of $\approx 2.15$ in long-wavelength limit, which is quite reasonable value for Lennard-Jones liquids. One should note, that $\gamma(k)$ is in fact a measure of coupling between the heat and viscous processes: for $\gamma=1$ one can describe heat and viscous 


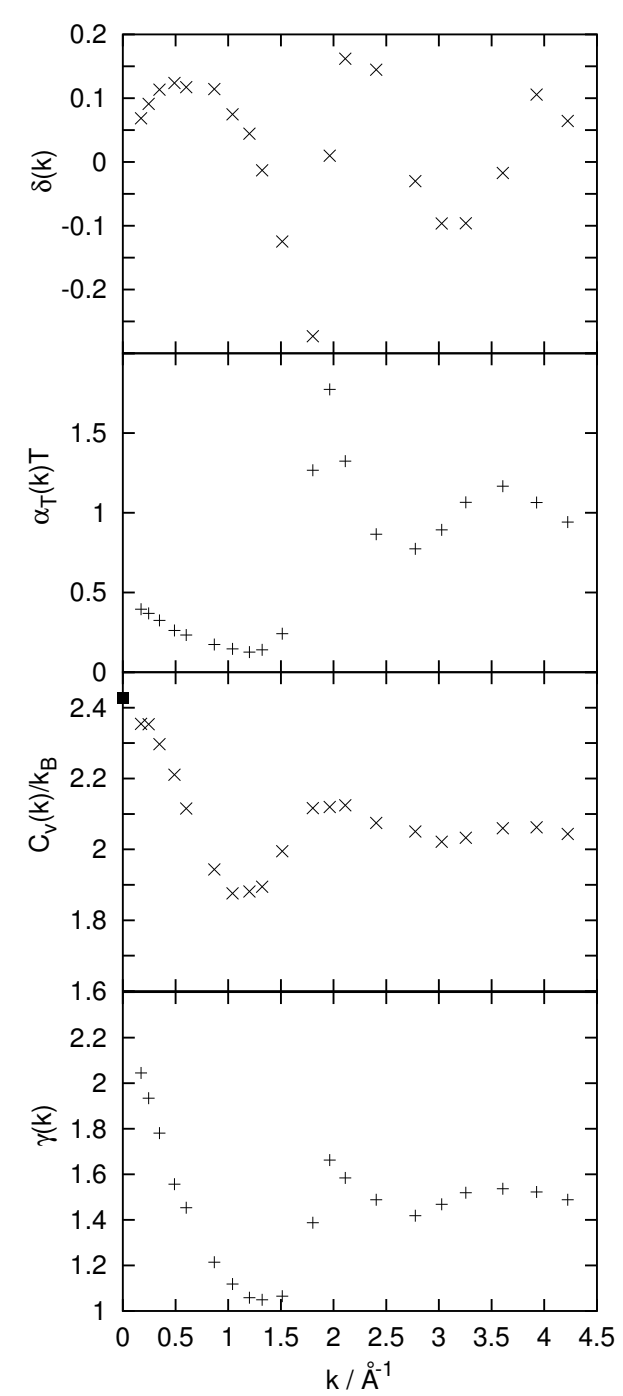

Figure 3. Generalized $k$-dependent thermodynamic quantities for $\mathrm{KrAr}$ mixture: the generalized dilatation $\delta(k)$; the generalized linear expansion coefficient $\alpha_{\mathrm{T}}(k)$; the generalized specific heat at constant volume $C_{\mathrm{V}}(k)$ (the filled box at $k=0$ corresponds to the value obtained directly in MD simulations); and the generalized ratio of specific heats $\gamma(k)$.

processes separately. That is why the longitudinal dynamics of liquid metals with the typical value of $\gamma$ being of the order $1.1-1.2$ is described pretty well within the viscoelastic theories. For Lennard-Jones liquids the coupling between the heat and viscous processes is usually rather strong and, for instance, only in the region of wavenumbers $k \approx 1.3 \AA^{-1}$ for the system considered the generalized ratio of specific heats $\gamma(k)$ approaches unity, so that the viscoelastic treatment could be successfully applied in this region.

The numerical results, obtained for the static averages

$$
\bar{\omega}_{4}^{t t}(k)=\frac{\left\langle\dot{J}_{t} \dot{J}_{t}^{*}\right\rangle}{\left\langle J_{t} J_{t}^{*}\right\rangle}, \quad \bar{\omega}_{4}^{t x}(k)=\frac{\left\langle\dot{J}_{t} \dot{J}_{x}^{*}\right\rangle}{\left\langle J_{x} J_{x}^{*}\right\rangle}, \quad \bar{\omega}_{4}^{x x}(k)=\frac{\left\langle\dot{J}_{x} \dot{J}_{x}^{*}\right\rangle}{\left\langle J_{x} J_{x}^{*}\right\rangle},
$$




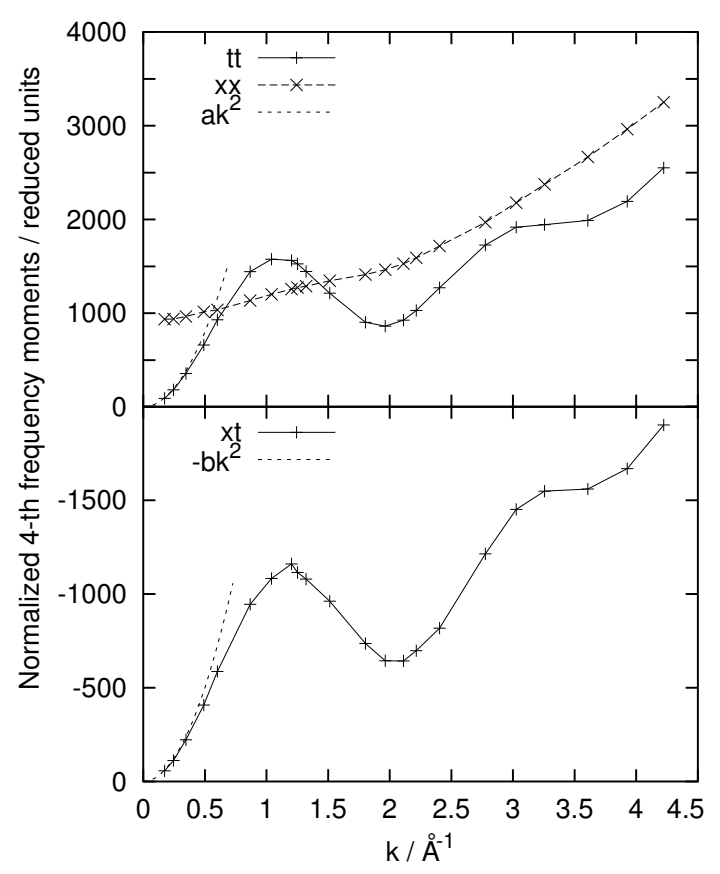

Figure 4. The ratio of fourth and second order frequency moments for the dynamic structure factor: density-density, $\bar{\omega}_{4}^{t t}(k)$; density-mass concentration, $\bar{\omega}_{4}^{t x}(k)$; mass concentration-mass concentration, $\bar{\omega}_{4}^{x x}(k)$. The hydrodynamic asymptotes are shown by short-dashed lines.

being directly connected with the ratio of fourth and second order frequency moments of relevant dynamic structure factors, are plotted in figure 4 . It is very important for the subsequent analysis that in the long-wavelength limit $\bar{\omega}_{4}^{x x}(k)$ tends to a non-zero constant, while the other static averages show clearly the $k^{2}$-behaviour in a small $k$ domain. We also note that the function $\bar{\omega}_{4}^{t t}(k)$ is often treated $[1,2]$ in connection with the dispersion law for sound excitations in the viscoelastic models. This is because the dynamic variable $\dot{J}_{t}(k, t)$ is proportional to the longitudinal component of stress tensor and in the limit $k \rightarrow 0$ the relevant static average defines the so-called high-frequency sound velocity $c_{\infty}$ :

$$
c_{\infty}^{2} k^{2}=\bar{\omega}_{4}^{t t}(k), \quad k \rightarrow 0 .
$$

Using this last relation we have estimated $c_{\infty} \approx 1210 \mathrm{~m} / \mathrm{s}$ for the studied $\mathrm{KrAr}$ mixture. In a similar way, the function $\bar{\omega}_{4}^{x x}(k)$ can be associated with the dispersion law for the propagating mass-concentration waves in long-wavelength limit, and the obvious feature of optic-like excitations with the nonzero frequency of propagation at $k=0$ can be easily obtained. It should be noted once again that this is a general property of a binary liquid, when the dissipation processes are neglected on such a level of description. 


\subsection{Time correlation functions}

In figures 5-7 the numerical results, obtained for time correlation functions of a binary $\mathrm{KrAr}$ liquid in MD simulations as well as within the GCM approach, are presented for several wavenumbers $k$. In these figures one can see how the GCM approach permits to reproduce six main TCFs of primary interest obtained in MD simulations on different spatial and time scales.
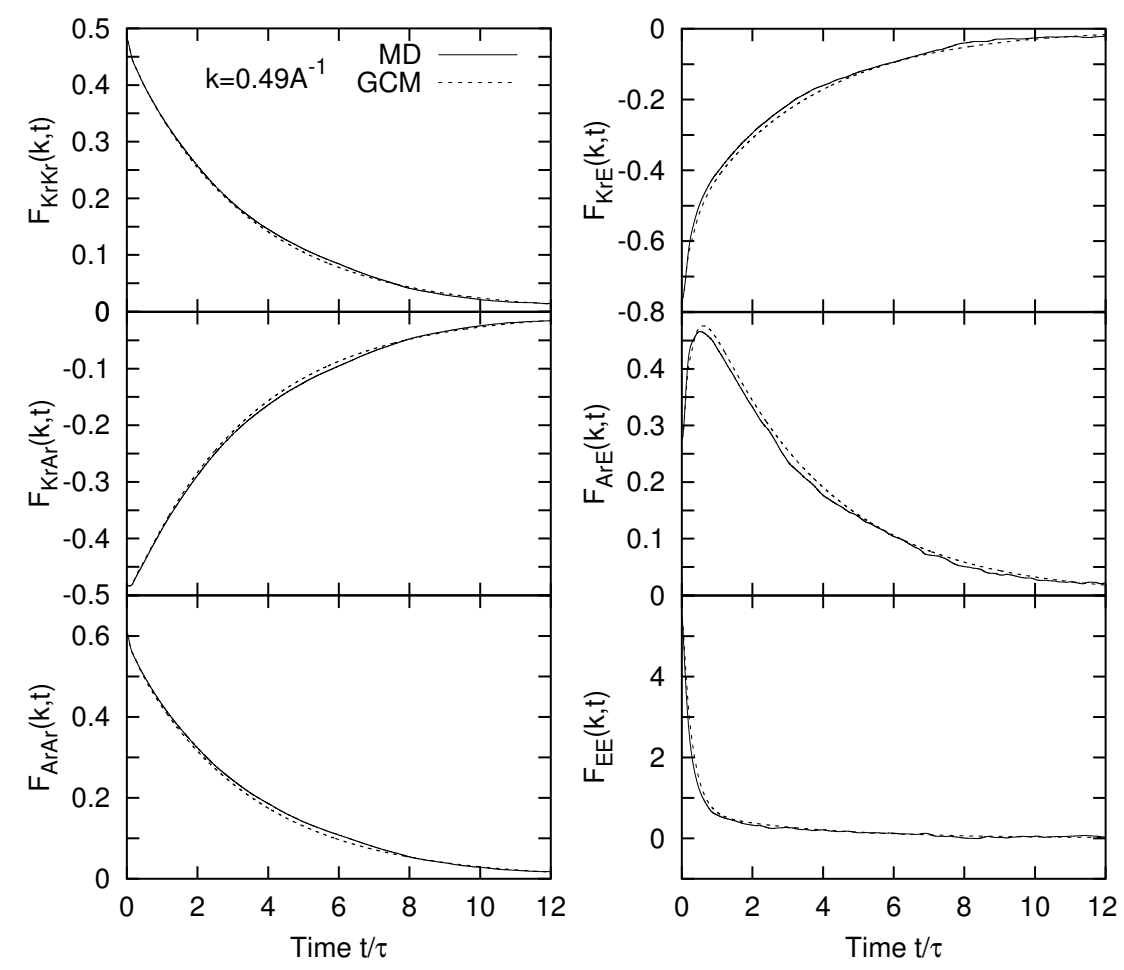

Figure 5. Time correlation functions obtained in MD simulations (solid lines) and their GCM counterparts (dashed lines) for a wavenumber $k=0.49 \AA^{-1}$ : the partial TCFs (on the left side) and the TCFs with the energy density (on the right side). The time scale is $\tau=4.598$ ps.

The GCM counterparts have been calculated with the help of theoretical expressions for time correlation functions (see, e.g., [12]), represented in this study as linear combinations of the eight contributions from the corresponding generalized collective excitations:

$$
\mathrm{F}_{i j}^{(\mathrm{GCM})}(k, t)=\sum_{\alpha=1}^{8} G_{\alpha}^{i j}(k) \mathrm{e}^{-z_{\alpha}(k) t},
$$

where $G_{\alpha}^{i j}(k)$ are the $k$-dependent amplitudes, describing the corresponding contribution of the collective mode $z_{\alpha}(k)$. Both the amplitudes $G_{\alpha}^{i j}(k)$ and the eigenvalues $z_{\alpha}(k)$ in general can be the complex quantities and are calculated from the eigenvalue problem for the generalized hydrodynamic matrix, generated in our case on the eight-variable basis set $\mathbf{A}^{(8)}(k)$. 

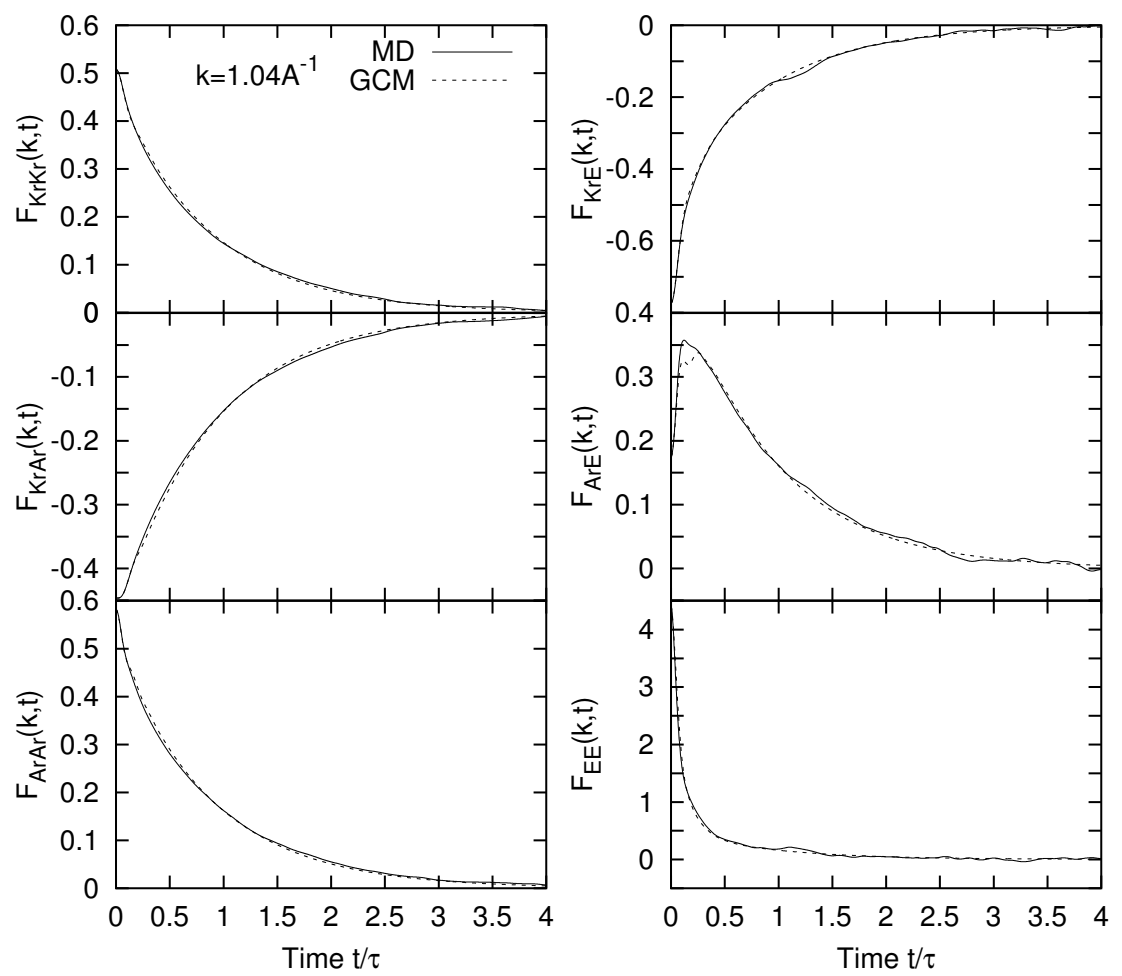

Figure 6. Time correlation functions obtained in MD simulations (solid lines) and their GCM counterparts (dashed lines) for a wavenumber $k=1.04 \AA^{-1}$ : the partial TCFs (on the left side) and the TCFs with the energy density (on the right side). The time scale is $\tau=4.598$ ps.

Comparing the curves, given in figures 5-7, it is seen that the GCM approach makes it possible to reproduce the time-dependence of MD-derived TCFs with high precision in a wide $k$ range considered. This conclusion is valid both for the partial TCFs, $\mathrm{F}_{\alpha \beta}(k, t)$ with $\alpha, \beta=\mathrm{Kr}$, Ar, and for the TCFs, constructed on the energy density variable, $\mathrm{F}_{\alpha \varepsilon}(k, t)$ with $\alpha=\mathrm{Kr}, \operatorname{Ar}$ and $\mathrm{F}_{\varepsilon \varepsilon}(k, t)$. The time scale of $\tau=$ 4.598 ps indicates that the range of time correlations at $k=0.49 \AA^{-1}$ (see figure 5) is of the order of $60 \mathrm{ps}$. For this reason, to provide a good convergence of the tails of time correlation functions, we have simulated the system for three smallest wavenumbers in the scale seven times longer.

The six time correlation functions shown in figures 5-7 define six main correlation times in a binary liquid:

$$
\tau_{i j}(k)=\frac{1}{F_{i j}(k, 0)} \int_{0}^{\infty} F_{i j}(k, t) \mathrm{d} t, \quad i, j=A, B, \varepsilon
$$

In this study the six correlation times were directly estimated from MD-derived TCFs for subsequent use in matrix elements of generalized hydrodynamic matrix $\mathbf{T}(k)$ generated on the basis set $\mathbf{A}^{(8)}(k)$. 

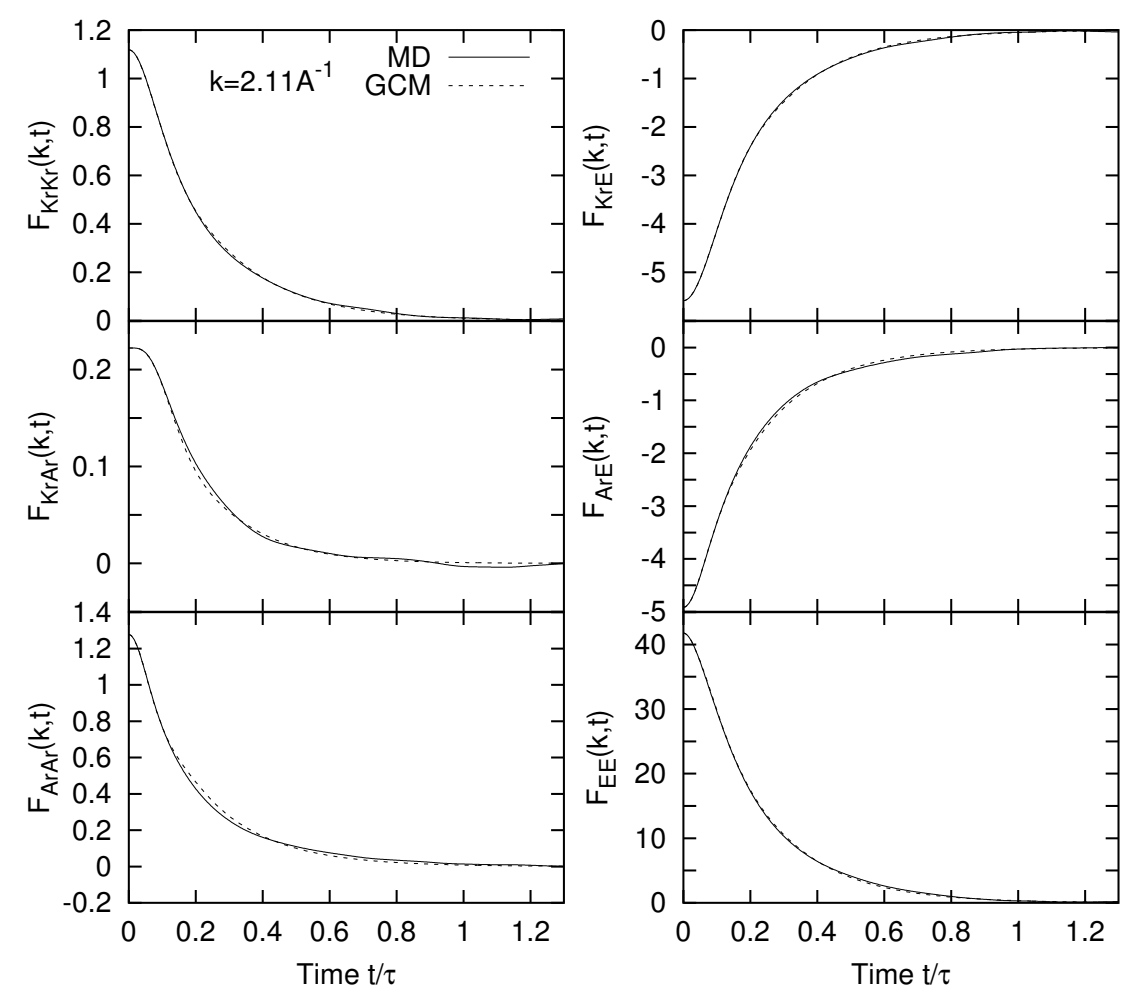

Figure 7. Time correlation functions obtained in MD simulations (solid lines) and their GCM counterparts (dashed lines) for a wavenumber $k=2.11 \AA^{-1}$ : the partial TCFs (on the left side) and the TCFs with the energy density (on the right side). The time scale is $\tau=4.598$ ps.

\section{Spectrum of collective excitations}

\subsection{Results for the eight-variable model}

Spectrum of propagating collective excitations (the imaginary and real parts of corresponding eigenvalues), obtained in our eight-variable GCM analysis, is shown in figure 8. The "plus" symbols in this figure correspond to the low-frequency heat waves with a propagation gap at small wavenumbers. Such a behavior is quite similar to the one observed previously for a heat wave branch in simple liquids. For the $\mathrm{KrAr}$ mixture there exists a rather wide propagation gap with a width $\approx 2.1 \AA^{-1}$, where the heat waves cannot propagate. Instead, two relaxing processes driven by the heat fluctuations exist in this region.

The purely real eigenvalues $d_{i}(k)$, describing three slowest relaxation-like processes in the system, are shown in figure 9. The hydrodynamic process of thermal diffusion is shown in figure 9 by filled circles. Note that when the wavenumber $k$ increases, the rapid growth of an inversed relaxation time for thermal diffusion is observed. This means that under such conditions the thermal diffusive process can play a crucial role in a small time scale only. Another relaxation process, caused by thermal fluctuations, has an extremely small relaxation time and is not shown in figure 9. At $k \simeq 2.1 \AA^{-1}$ the relaxation times of both thermal relaxation processes 


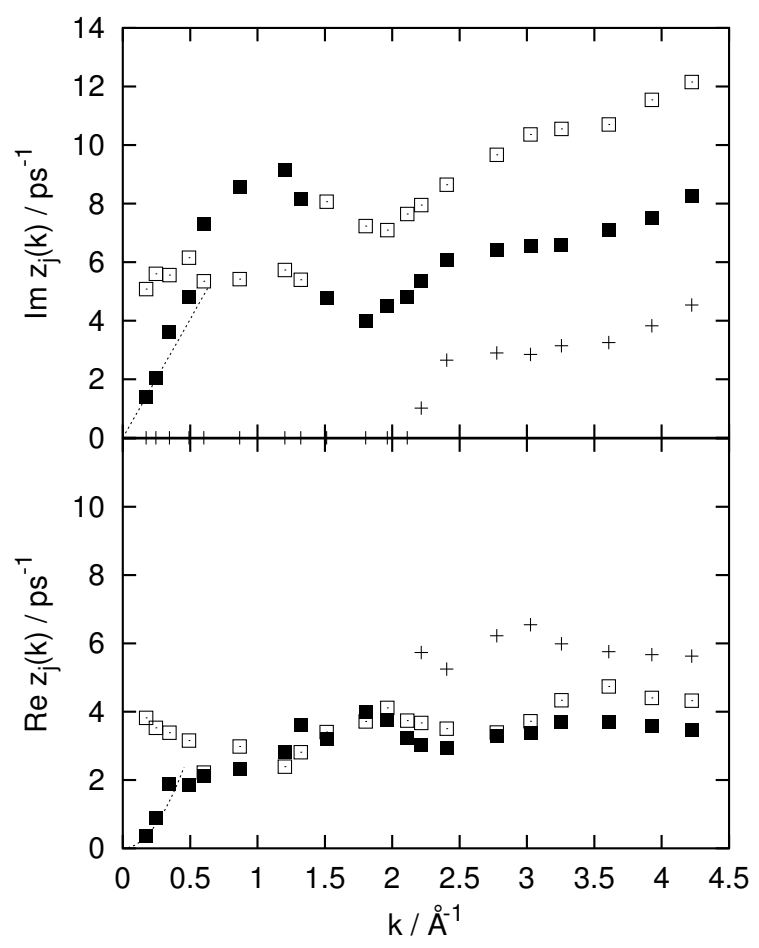

Figure 8. Imaginary and real parts of propagating eigenvalues, obtained for the eight-variable set $\mathbf{A}^{(\boldsymbol{8})}(k)$. The small- $k$ asymptotes are shown for the generalized sound excitations by dotted lines.

become close in magnitude and the heat waves emerge in the liquid.

Two branches of propagating excitations, existing in the whole $k$ range studied, are shown in figure 8 by filled and open boxes. One should note, that for $k>$ $0.5 \AA^{-1}$ both branches have nearly the same damping coefficients, while in the small wavenumbers limit the branch shown by open boxes tends to a finite nonzero damping coefficient. This is a specific feature of kinetic-like collective modes $[11,12]$. The branch shown by filled boxes displays almost linear $k$ dependence for imaginary part of the eigenvalue with the slope $c=808 \mathrm{~m} / \mathrm{s}$, while its real part for $k<$ $0.3 \AA^{-1}$ behaves almost proportional to a $k^{2}$ which is typical of sound excitations. Hence, we have sufficient reasons to argue that the branches shown by filled and open boxes describe the generalized sound excitations and the kinetic optic-like propagating modes, respectively. The discussion of this subject will be continued in the next subsection within the simplified models of the longitudinal dynamics giving additional insight into the mode formation mechanism.

Beyond the hydrodynamic region two relaxing processes become very important. The first one (see the eigenvalue shown by open triangles in figure 9) has a finite nonzero relaxation time in the long-wavelength region and obviously does not significantly contribute to the shape of spectral functions in this domain of wavenumbers. The short-dashed curve with $k^{2}$-dependence very well fits (see figure 9 ) the behaviour of this relaxing mode for $k<0.6 \AA^{-1}$. We note that similar kinetic relaxing process, associated with the structural relaxation phenomenon, has been identified in sim- 


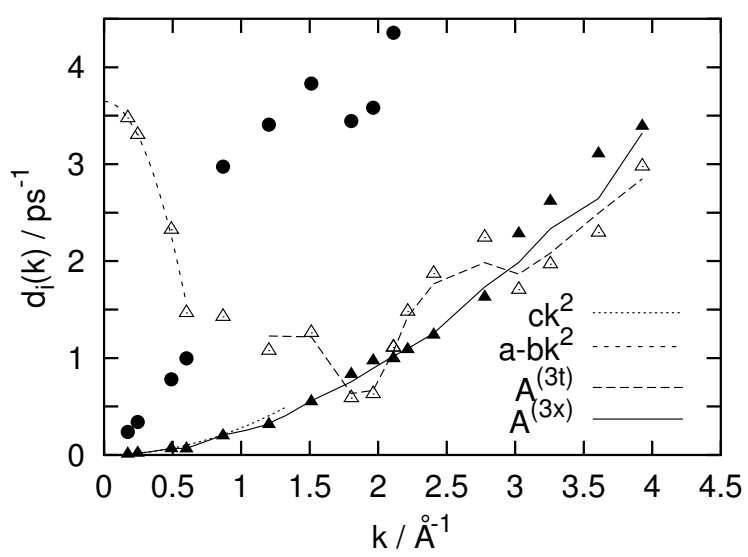

Figure 9. Purely real eigenvalues $d_{i}(k)$, describing the relaxation processes in a binary KrAr liquid, obtained within the GCM approach: symbols - results for the basis set $\mathbf{A}^{(8)}(k)$; solid and long dashed lines - for the separated three-variable sets $\mathbf{A}^{(3 t)}$ and $\mathbf{A}^{(3 x)}$. The small- $k$ asymptotes are shown for the hydrodynamic diffusion mode by dotted line and for the generalized viscous relaxation mode by short-dashed line.

ple liquids $[5,6,20]$. In particular, it was found that the corresponding eigenvalues behave in small $k$ range as follows:

$$
d_{\mathrm{str}}(k)=\frac{c_{\infty}^{2}-c_{\mathrm{s}}^{2}}{D_{\mathrm{L}}}-D_{\mathrm{L}} k^{2}-(\gamma-1) A k^{2},
$$

where $D_{\mathrm{L}}$ is longitudinal viscosity, $c_{\mathrm{s}}$ denotes an adiabatic sound velocity, and $A$ is some constant defined by thermodynamic quantities and transport coefficients. An important point is that, in simple liquids, the structural relaxation process becomes the dominant one beyond the hydrodynamic region and makes the main contribution to the central peak of dynamic structure factor $[5,6,20]$. In the case of a binary liquid considered one can see in figure 9 that two relaxation processes have nearly comparable relaxation times for $k>1.5 \AA^{-1}$. In addition to the structural relaxation mode, there is another collective excitation with the purely real eigenvalue, shown in figure 9 by filled triangles. Taking into account the $k^{2}$-asymptote of the mentioned eigenvalue in a small $k$ domain (shown by dotted line), one can conclude that this second relaxation process is just associated with the mutual diffusion. In the next subsection we discuss such a conclusion by considering two different subsets of dynamic variables for the analysis of time correlation functions and the collective mode spectrum in order to separate the relaxation processes of different origin.

\subsection{Simplified models of the longitudinal dynamics}

One of the advantages of the GCM approach consists in a possibility to perform an additional study for separated subsets of dynamic variables. In comparison with the results found for the basis set of dynamic variables, this allows us to establish the physical origin of different modes in the spectrum of collective excitations and to 
determine the dominant dynamic processes on various spatial and time scales. Let us consider two separated three-variable subsets, describing the uncoupled dynamics of the total density and mass-concentration fluctuations:

$$
\mathbf{A}^{(3 \alpha)}=\left\{n_{\alpha}, J_{\alpha}, \dot{J}_{\alpha}\right\}, \quad \alpha=t, x .
$$

Note that the joint set $\mathbf{A}^{(6)}(k)$, which just includes the dynamic variables of both these two subsets, forms an appropriate six-variable basis for the study of longitudinal dynamics in the viscoelastic approximation. It is also obvious that for the separated subsets, the $t-x$ cross-correlations are neglected. However, in the case of weak coupling one can expect that the solutions of two $3 \times 3$ secular equations, generated for subsets (10), will produce the results being very close to the eigenvalues, obtained for the eight-variable dynamic model (8). This would permit to identify the origin of different branches in the spectrum of collective excitations and to estimate the role of mode-coupling effects. Similar analysis, based on the separated subsets of dynamic variables, has been successfully applied in our previous study of transverse collective excitations in binary liquids [8,9] and allowed us to arrive at the unambiguous conclusions concerning the role of transverse optic-like excitations in binary mixtures.

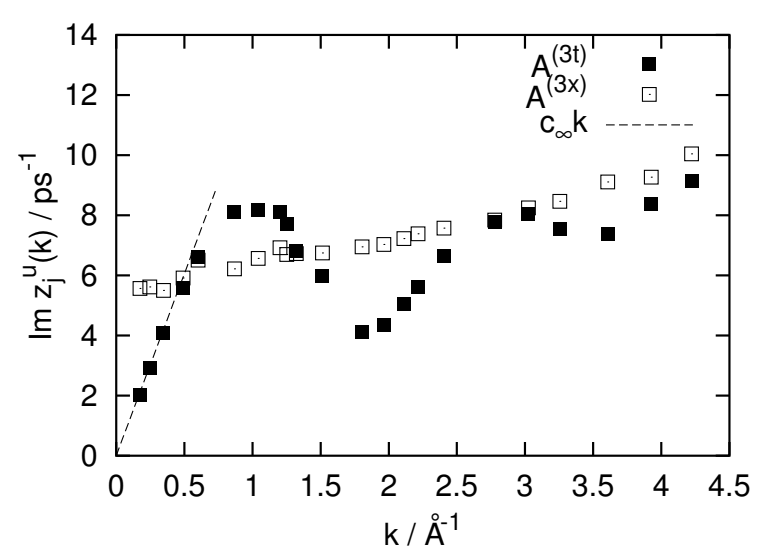

Figure 10. Imaginary parts of the propagating eigenvalues, obtained for the separated three-variable subsets $\mathbf{A}^{(3 t)}$ and $\mathbf{A}^{(3 x)}$. The small- $k$ asymptote is shown for the sound excitations by long-dashed line.

The results of our calculations, obtained for the dispersions of propagating collective modes on the separated subsets $\mathbf{A}^{(3 t)}(k)$ (filled boxes) and $\mathbf{A}^{(3 x)}(k)$ (open boxes), are shown in figure 10. One can immediately see the similarity of these dispersion curves with the functions presented in figure 4 (upper part). Moreover, comparing the linear slope of the lower branch in figure 10 in a small $k$ domain with the behaviour of the relevant fourth order frequency moment, we see that the separated basis set $\mathbf{A}^{(3 t)}(k)$ allows us to obtain the sound-like excitations with the propagating velocity $c_{\infty}$ typical of the elastic medium. Thus, the cross-correlations with the heat and mass-concentration fluctuations, which are correctly taken into account within the eight-variable model $\mathbf{A}^{(8)}(k)$, renormalize the sound velocity to 
the value of $c=808 \mathrm{~m} / \mathrm{s}$, which is in quite good agreement with the adiabatic speed of sound $c_{\mathrm{s}}=755 \pm 35 \mathrm{~m} / \mathrm{s}$, estimated from the functions $\gamma(k)$ and $\kappa_{\mathrm{T}}(k)$ (see figure 3 ) in the limit $k \rightarrow 0$.

Purely real eigenvalues, calculated for the simplified dynamical models on the separated subsets, are shown by long-dashed and solid lines in figure 9 . One can see that in complete agreement with the case of simple liquids the eigenvalue, describing structural relaxation has a minimum at the position of the main peak of total static structure factor $k_{\mathrm{p}}$. Comparing the results presented in figure 9 , we can conclude that the low-lying relaxing mode shown by filled triangles is directly connected with the mutual diffusion process.

\subsection{Partial current spectral functions}

Quite common practice in the literature is to use for the study of collective excitations different spectral functions, which can be obtained in the scattering experiments or known from MD simulations. These functions are defined as the Fourier-transforms of corresponding time correlation functions. In this subsection we use such an approach for the estimations of the dispersion curves for the high- and low-frequency branches of propagating modes. This can be done from the location of maxima positions for the partial 'current-current' spectral functions $C_{\alpha \alpha}(k, \omega)$, being simply connected to the partial dynamic structure factors $S_{\alpha \alpha}(k, \omega)$,

$$
C_{\alpha \alpha}(k, \omega)=\frac{m_{\alpha}^{2} \omega^{2}}{k^{2}} S_{\alpha \alpha}(k, \omega)
$$

with $\alpha=\mathrm{Kr}$, Ar. Our aim herein is to compare the dispersions obtained with the spectrum of propagating collective modes, calculated within the GCM approach.

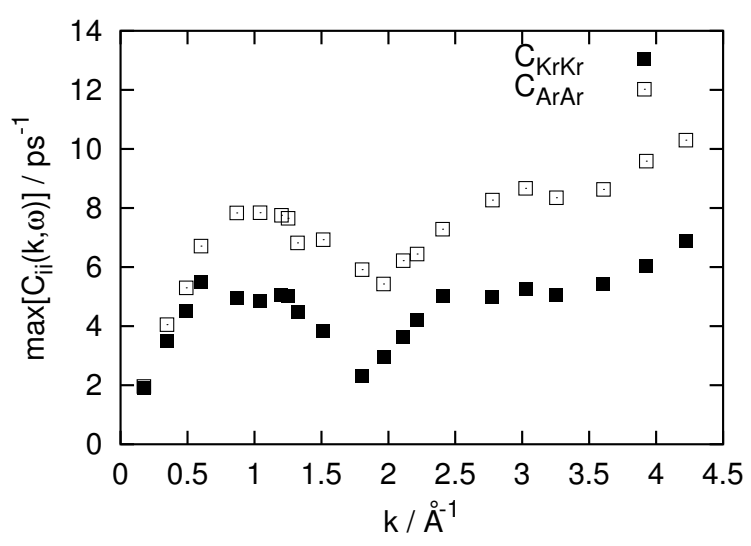

Figure 11. Maxima positions of the partial spectral functions $C_{\alpha \alpha}(k, \omega)$ with $\alpha=\mathrm{Kr}, \mathrm{Ar}$ as functions of wavenumber $k$.

In figure 11 we show the $k$-dependence of peak positions of the partial current spectral functions $C_{\mathrm{KrKr}}(k, \omega)$ and $C_{\mathrm{ArAr}}(k, \omega)$. One can see that the two branches basically agree with the GCM spectrum in the region $k>0.7 \AA^{-1}$. We note that, as it was found in our study of the transverse dynamics $[8,9]$, beyond the hydrodynamic 
region the partial character of the dynamics prevails. The same conclusion has been recently made for the longitudinal dynamics in binary liquids [13], so that the results presented in figure 11 are in agreement with our previous studies. However, for smaller wavenumbers the method of estimation for dispersion curves from the peak positions of partial current spectral functions $C_{\alpha \alpha}(k, \omega)$ produces a merger of the two branches exactly as it was reported in [15-18].

Let us discuss this contradiction more in detail. One should note that the numerical scheme for dispersion estimations from the peak positions of $C_{\alpha \alpha}(k, \omega)$ is applied to the dynamics of binary liquids in complete analogy with the method widely used in collective dynamics of simple liquids. However, this method: (i) does not take into account that in binary liquids there exist at least two different propagating excitations, which could be observed in spectral functions under certain conditions only (e.g., the frequencies of the modes are well separated, the modes are not overdamped, and the mode contributions for both modes are nearly of the same order, etc); (ii) is based on the simplified assumption that the characteristic frequency of a propagating mode determines alone the maximum position in the spectral functions; and, therefore, (iii) can be applied only for very rough estimates of the dispersion dependencies.
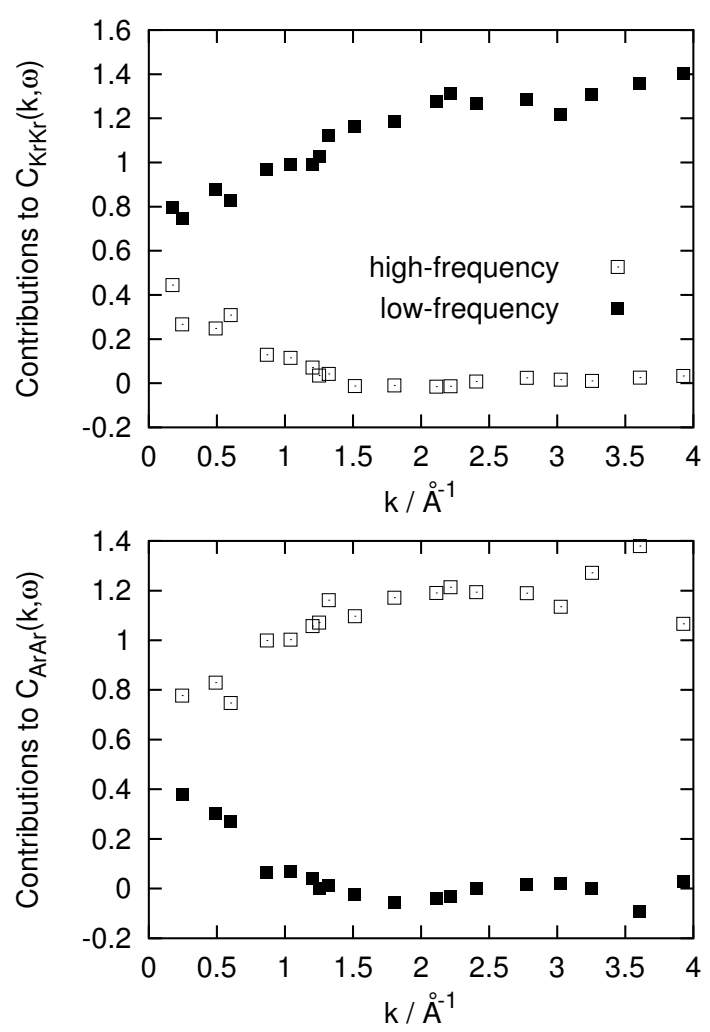

Figure 12. Amplitudes of the mode contributions from the optic-like (highfrequency) and generalized sound (low-frequency) propagating excitations to the spectral function $C_{\mathrm{KrKr}}(k, \omega)$ (on the top) and $C_{\mathrm{ArAr}}(k, \omega)$ (on the bottom) as functions of wavenumber $k$. 
In a binary liquid, a sufficient difference between two branches of propagating collective excitations appears in a small $k$ domain only, where the optic-like modes have a finite damping coefficient even in $k \rightarrow 0$ limit. This results in a rather flat Lorentzian-like contribution to spectral functions with a weakly developed peak structure, while the sound excitations in that limit form an extremely well-pronounced Lorentzian with a well observed peak structure at sound frequencies. Therefore, the optic-like modes contribution can be mostly seen in the shape of spectral function as some shoulders, which are difficult to identify as real peaks. That is why the spectral method, widely used in the literature for the study of dispersion laws and based on numerical estimations of the peak positions in spectral functions, cannot be considered suitable for identification of optic-like excitations in binary liquids.

To complete this section, in figure 12 we have show the amplitudes of mode contributions from the high- and low-frequency branches to the partial spectral functions $C_{\alpha \alpha}(k, \omega)$, calculated within the GCM approach (definitions can be found in $[9,13])$. It is clearly seen that for $k>1 \AA^{-1}$ the partial picture in the longitudinal dynamics dominates and the main contribution to each partial spectral function is mainly determined by one propagating mode of certain type. We recall in this connection that for wavenumbers beyond the hydrodynamic region the spectrum of propagating modes could be very well reproduced $[8,9]$ within simplified models of the partial dynamics. However, in small $k$ domain the mode contributions from the two propagating branches to $C_{\alpha \alpha}(k, \omega)$ become comparable (see figure 12). And with comparable amplitudes the optic-like excitations have no chances to be seen as an extra maximum in partial spectral functions. In general, the results, presented in figure 12, nicely illustrate the crossover in the longitudinal dynamics from the collective behavior to the partial one, when wavenumber $k$ increases.

\section{Conclusions}

We conclude by making the following remarks:

(i) In this paper the collective excitation spectrum of a binary equimolar LennardJones KrAr liquid at the temperature $116 \mathrm{~K}$ has been studied within the GCM approach based on the eight-variable dynamic model. Three branches of propagating excitations, corresponding to the generalized sound, the optic-like modes, caused by mass-concentration fluctuations, and the low-frequency heat waves, have been identified. In the region $k<0.3 \AA^{-1}$, almost linear dispersion with the sound velocity of $c_{\mathrm{s}}=808 \mathrm{~m} / \mathrm{s}$ is found for the generalized sound excitations, and this result is in good agreement with another estimated value of $c_{\mathrm{s}}=755 \pm 35 \mathrm{~m} / \mathrm{s}$, obtained independently. The dispersion of high-frequency optic-like excitations tends to a finite frequency $\omega_{\text {opt }} \simeq 5 \mathrm{ps}^{-1}$ at $k \rightarrow 0$ but with the nonzero damping coefficient $\sigma_{\mathrm{opt}} \simeq 4 \mathrm{ps}^{-1}$. For the low-frequency heat waves, a propagation gap with the width of $2.1 \AA^{-1}$ is obtained;

(ii) In agreement with the recent findings for simple liquids, it is shown that the role of structural relaxation process in a binary liquid rapidly increases beyond the 
hydrodynamic region, while the thermal diffusion becomes almost irrelevant in the collective dynamics for $k>1 \AA^{-1}$. However, the specific feature of a binary liquid is the existence (in addition to the structural relaxation) of another relaxation process, connected with mutual diffusion, with comparable relaxation time in the region of the main peak of total static structure factor;

(iii) It is shown that the analysis of the dispersion laws for propagating modes, based on the numerical estimates of maxima positions in the partial current spectral functions $C_{\alpha \alpha}(k, \omega)$, leads to the dispersion of high- and low-frequency branches with their merger in long-wavelength region. This is in contrast with the results, obtained for generalized collective excitations within the GCM approach. We have explained the reasons for such contradiction and argued the limitations of the spectral method widely used in the literature for the study of dispersion laws;

(iv) It is shown that the partial picture of the dynamics dominates in a binary liquid for large wavenumbers, in particular for a Lennard-Jones KrAr liquid studied for $k>1 \AA^{-1}$, while for smaller $k$ values, the contributions from the two propagating branches to $C_{\alpha \alpha}(k, \omega)$ become comparable. This gives a good example of the crossover in longitudinal dynamics from the partial behavior at larger wavenumbers to a picture where the collective dynamics with the well-defined sound and optic-like excitations prevails in a small- $k$ region. We argue that since the optic-like excitations belong to kinetic-like modes with a finite damping in $k \rightarrow 0$ limit, its contributions to the partial spectral functions $C_{\alpha \alpha}(k, \omega)$ are very difficult to identify using the spectral method alone.

\section{Acknowledgements}

I.M. thanks the Fonds zur Förderung der wissenschaftlichen Forschung (Austria) for financial support under Project No. P15247.

\section{References}

1. Boon J.-P., Yip S. Molecular Hydrodynamics. New-York: McGraw-Hill, 1980.

2. Hansen J.-P., McDonald I.R. Theory of Simple Liquids. London: Academic, 1986.

3. Scopigno T., Balucani U., Ruocco G., Sette F. // J. Phys.: Cond. Matt., 2000, vol. 12, p. 8009.

4. Scopigno T., Ruocco G., Sette F., Viliani G. // Phys. Rev. E, 2002, vol. 66, p. 031205.

5. Bryk T., Mryglod I. // Phys. Rev. E, 2001, vol. 63, p. 051202.

6. Bryk T., Mryglod I. // Phys. Rev. E, 2001, vol. 64, p. 032202.

7. Bosse J., Jacucci G., Ronchetti M., Schirmacher W. // Phys. Rev. Lett., 1986, vol. 57, p. 3277.

8. Bryk T., Mryglod I. // Phys. Rev. E, 2000, vol. 61, p. 2088.

9. Bryk T., Mryglod I. // J. Phys.: Cond. Matt., 2000, vol. 12, p. 6063.

10. de Schepper I.M., Cohen E.G.D., Bruin C., van Rijs J.C., Montfrooij W., de Graaf L.A. // Phys. Rev. A, 1998, vol. 38, p. 271.

11. Mryglod I.M., Omelyan I.P., Tokarchuk M.V. // Mol. Phys., 1995, vol. 84, p. 235.

12. Mryglod I.M. // Condens. Matt. Phys., 1998, vol. 4(16), p. 753. 
13. Bryk T., Mryglod I. // J. Phys.: Cond. Matt., 2002, vol. 14, p. L445.

14. Bryk T., Mryglod I., Kahl G. // Phys. Rev. E, 1997, vol. 56, p. 2903.

15. Enciso E., Almarza N.G., Dominguez P., Gonzalez M.A., Bermejo F.J. // Phys. Rev. Lett., 1995, vol. 74, p. 4233.

16. Fernandez-Perea R., Alvarez M., Bermejo F.J., Verkerk P., Roessli B., Enciso E. // Phys. Rev. E, 1998, vol. 58, p. 4568.

17. Anento N., Padro J.A. // Phys. Rev. E, 2001, vol. 64, p. 021202.

18. Gonzalez D.J., Gonzalez L.E., Lopez J.M., Stott M.J. // Europhys. Lett., 2003, vol. 62, p. 42.

19. March N.H., Tosi M.P. Atomic Dynamics in Liquids. Macmillan Press, 1976.

20. Bryk T., Mryglod I. // J. Phys.: Cond. Matt., 2001, vol. 13, p. 1343.

\title{
Повздовжня динаміка в ленард-джонсівській бінарній рідині: кросовер від гідродинаміки до молекулярного режиму
}

\author{
Т.Брик, І.Мриглод \\ Інститут фізики конденсованих систем НАН України, \\ 79011 Львів, вул. Свєнціцького, 1 \\ Отримано 21 лютого 2004 р.
}

Повздовжня колективна динаміка в еквімолярній ленард-джонсівській суміші $\mathrm{KrAr}$ детально досліджується в широкій області просторових та часових масштабів. Комбінуючи симуляції методом молекулярної динаміки та аналітичний підхід узагальнених колективних мод, ми обчислили спектр узагальнених колективних збуджень та проаналізували основні динамічні процеси, що визначають головні вклади в часові кореляційні функції у різних областях - починаючи від гідродинамічної границі та аж до області так званого молекулярного режиму. Встановлено походження колективних пропагаторних мод і особливості їх дисперсії в гідродинамічній області та поза нею. Показано, що структурна релаксація та процеси, пов'язані з взаємною дифузією частинок, в основному визначають центральний пік повного динамічного структурного фактора поза гідродинамічною областю. Закони дисперсії, отримані в аналітичному підході, порівнюються з дисперсійними кривими, визначеними з положень максимумів спектральних функцій для парціальних потоків. Обговорюється різниця між цими двома наборами чисельних результатів.

Ключові слова: колективна динаміка, бінарна суміш, колективне збудження, структурна релаксація

PACS: 05.20.Jj, 61.20.Ja, 61.20.LC 